\title{
QUALIDADE DE VIDA DE PACIENTES DIAGNOSTICADOS COM ESPONDILITE ANQUILOSANTE: REVISÃO INTEGRATIVA
}

\author{
João Paulo Monteiro Soares ${ }^{1}$, Ana Gabriela de Sousa Costa ${ }^{1}$, Nádia Vicência \\ do Nascimento Martins ${ }^{2}$, Ana Emília Gomes Macedo ${ }^{3}$ \\ ${ }^{1}$ Curso de Medicina, Centro de Ciências Biológicas e da Saúde, Universidade do Estado do \\ Pará, Santarém, Pará, Brasil \\ 2 Doutora em Enfermagem, Prof ${ }^{a}$ no Centro de Ciências Biológicas e da Saúde, Universidade \\ do Estado do Pará, Santarém, Pará, Brasil. \\ ${ }^{3}$ Mestra em Bioengenharia, Prof ${ }^{a}$ no Centro de Ciências Biológicas e da Saúde, Universidade \\ do Estado do Pará, Santarém, Pará, Brasil.
}

Email para correspondência: jpmonteirosoares2017@gmail.com

\begin{abstract}
Resumo
A pesquisa objetivou avaliar a influência da Espondilite Anquilosante na qualidade de vida dos pacientes e o impacto de possíveis abordagens, por meio de revisão integrativa. Utilizou-se as bases de dados Scientific Electronic Library Online, Biblioteca Virtual em Saúde, Literatura Latino-Americana e do Caribe em Ciências da Saúde e PubMed. Foram analisados artigos publicados no período de 2010 a 2020 e selecionados conforme os critérios de inclusão e exclusão preestabelecidos. A amostra final foi constituída por vinte artigos. Observou-se que a produtividade, a mobilidade, a dor articular e o envolvimento do quadril foram as principais debilitações encontradas. Jovens adultos do sexo masculino permanecem sendo a população mais afetada, bem como o tratamento medicamentoso sendo a terapêutica mais utilizada, com destaque para o uso de Inibidores do Fator de Necrose Tumoral, Medicamentos Modificadores do Curso da Doença e Anti-Inflamatórios Não Esteroides. Entretanto, observou-se um uso crescente de intervenções não medicamentosas, como exercícios aeróbicos e fisioterapia, com ênfase na abordagem multiprofissional e na melhoria da qualidade de vida. $\mathrm{O}$ acometimento por Espondilite Anquilosante envolve sérios prejuízos funcionais aos pacientes acometidos, no entanto, a adoção de um esquema de tratamento multiprofissional e eficaz amplia a qualidade de vida dos pacientes.
\end{abstract}

Palavras-chave: Espondilite anquilosante. Qualidade de vida. Reumatologia. Espondiloartrite. Perfil de impacto da doença.

\begin{abstract}
The research aimed to evaluate the influence of Ankylosing Spondylitis on the quality of life of patients and the impact of possible approaches, through an integrative review. The Scientific Electronic Library Online, Virtual Health Library, Latin American and Caribbean Literature in Health Sciences and PubMed databases were used. Articles published in the period from 2010 to 2020 were analyzed and selected according to the pre-established inclusion and exclusion criteria. The final sample consisted of twenty articles. It was observed that productivity, mobility, joint pain, and hip involvement were the main weaknesses found. Young male adults remain the most affected population, as well as drug
\end{abstract}


treatment being the most widely used therapy, with emphasis on the use of Tumor Necrosis Factor Inhibitors, Disease Modifying Drugs and Non-Steroidal AntiInflammatory Drugs. However, there has been an increasing use of non-drug interventions, such as aerobic exercise and physical therapy, with an emphasis on the multiprofessional approach and improving the quality of life. Affection by Ankylosing Spondylitis involves serious functional impairments to the patients, however, the adoption of a multidisciplinary and effective treatment scheme increases their quality of life.

Keywords: Spondylitis, ankylosing. Quality of life. Rheumatology. Spondyloarthritis. Sickness impact profile.

\section{Introdução}

A espondilite anquilosante (EA) é a principal representante do grupo das espondiloartrites (EpA), doenças caracterizadas pela dor axial inflamatória, sacroílite e soro negatividade para o fator reumatoide (SAMPAIO-BARROS et al., 2013). O diagnóstico ocorre por meio de exame clínico e de imagem, sendo que exames laboratoriais, como testes da velocidade de sedimentação das hemácias (VHS) e proteína C Reativa, contribuem para indicar o estágio da doença (COMISSÃO DE ESPONDILOARTRITES, 2012).

A EA não apresenta cura, havendo apenas perspectiva de atenuação da progressão do quadro por meio de tratamento medicamentoso ou não. A doença, à princípio, manifesta-se por dores na coluna, podendo progredir para redução de expansibilidade torácica e limitação de movimentos (COMISSÃO DE ESPONDILOARTRITES, 2012). Associado a isso, a doença afeta outros sistemas como o renal, neurológico e pulmonar, comprometendo, assim, a qualidade de vida do paciente (RODRIGUES et al., 2012).

O tratamento farmacológico objetiva a redução do quadro inflamatório (BRASIL, 2018), devendo ser associados ao recurso nutricional como forma de ter seus efeitos potencializados e de minimizar as consequências extra esqueléticas da doença (ARAÚJJ, 2017), enquanto a fisioterapia contribui para a atenuação dos efeitos progressivos da EA (ARAÚJO et al., 2015).

Nesse sentido, é necessário compreender os efeitos da doença, associando-os ao perfil epidemiológico. Dessa forma, pode-se apurar quais os impactos do tratamento sobre a forma de progressão, além de verificar a disponibilidade e acessibilidade dos recursos terapêuticos para o público. Assim sendo, o objetivo da pesquisa é avaliar a influência da EA na qualidade de vida dos pacientes e o impacto de possíveis abordagens. 


\section{Metodologia}

A realização da pesquisa, que consiste em uma revisão integrativa, adotou seis etapas, descritas a seguir, propostas por Botelho; Cunha; Macedo (2011), objetivando contemplar todos os aspectos necessários para esse modelo de estudo.

\subsection{Primeira Etapa: identificação do tema e seleção da questão da pesquisa}

A partir da análise da Espondilite anquilosante e suas consequências, foi definido o problema e mediante a ele, as questões norteadoras: "Quais as principais complicações da Espondilite Anquilosante e suas interferências no cotidiano dos pacientes?", "qual o perfil da parcela mais afetada pela doença?" e "de que forma o tratamento para Espondilite Anquilosante influencia na qualidade de vida?".

Para responder às questões, foi realizada uma revisão integrativa, utilizando como descritores ("Espondilite Anquilosante" $O R$ "Espondilitis Anquilosante" $O R$ "Ankylosing Spondylitis") AND ("qualidade de vida" $O R$ "calidad de vida" $O R$ welfare), tendo como bases de dados Scientific Electronic Library Online (SciELO), Biblioteca Virtual em Saúde (BVS), Literatura LatinoAmericana e do Caribe em Ciências da Saúde (LILACS) e PubMed.

$\mathrm{Na}$ busca realizada na base de dados BVS, houve aplicação de filtros referentes ao assunto principal: Espondilite anquilosante; qualidade de vida; depressão; avaliação da deficiência; nível de saúde; terapia por exercício, exercício físico; indicadores básicos de saúde; efeitos psicossociais da doença.

\subsection{Segunda Etapa: estabelecimento dos critérios de inclusão e exclusão}

Foram incluídas produções completas no âmbito de artigos científicos, publicados no período de 2010 a outubro de 2020, disponíveis online e gratuitamente, nos idiomas português, inglês e espanhol. Como fatores para exclusão, foram englobadas produções na modalidade de dissertação de mestrado e tese de doutorado, resumos simples e expandidos, estudos de caso e revisões.

As etapas metodológicas seguidas para a seleção dos artigos com base nos critérios predeterminados estão representadas abaixo, na Figura 1. 


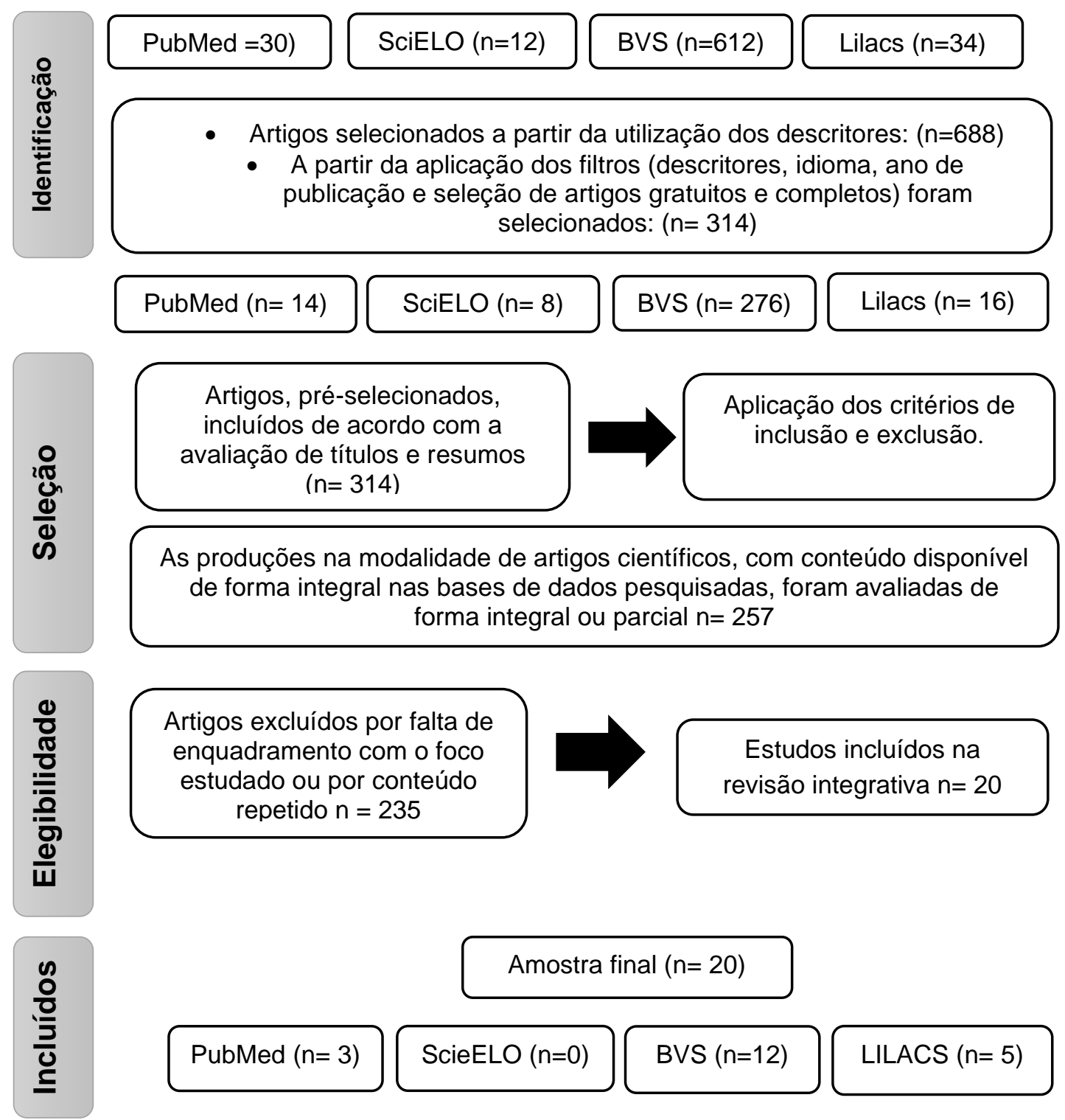

Figura 1 - Fluxograma referente às etapas metodológicas para seleção de artigos Fonte: Elaborado pelos autores, 2021.

\subsection{Terceira Etapa: identificação dos estudos pré-selecionados e selecionados}

As produções pré-selecionadas foram submetidas a um processo classificatório, observando-se título, resumo e palavras-chave, sendo realizada uma análise com base nos critérios de inclusão e exclusão para determinar os artigos selecionados. 


\subsection{Quarta Etapa: categorização dos estudos selecionados}

Foi elaborada uma Matriz de Síntese com o auxílio do software Microsoft Exce/® 2010, como forma de organizar os artigos selecionados para melhor direcionamento e extração de informações acerca das pesquisas. A matriz foi estruturada da seguinte forma: número (como forma de identificação e ordenação dos artigos), título do artigo, bases de dados, ano de publicação, país, autores e periódico (Quadro 1).

\subsection{Quinta Etapa: análise e interpretação dos resultados}

Após a categorização dos resultados, foi realizada análise crítica sobre os artigos, objetivando responder às questões propostas pela pesquisa, descritas no tópico 2.1.

\subsection{Sexta Etapa: apresentação da revisão/síntese do conhecimento}

Mediante a interpretação dos resultados, esses foram descritos detalhadamente, seguindo as etapas propostas, contemplando o objetivo da pesquisa.

\section{$3 \quad$ Resultados e discussão}

Quadro 1 - Descrição dos artigos selecionados na amostra

\begin{tabular}{|c|c|c|c|c|}
\hline № & TÍTULO DO ARTIGO & $\begin{array}{l}\text { BASES } \\
\text { DE } \\
\text { DADOS }\end{array}$ & $\begin{array}{c}\text { AUTOR/ } \\
\text { ANO/ PAÍS }\end{array}$ & PERIÓDICO \\
\hline N1 & $\begin{array}{l}\text { A three-week } \\
\text { multidisciplinary in-patient } \\
\text { rehabilitation programme } \\
\text { had positive long-term } \\
\text { effects in patients with } \\
\text { ankylosing spondylitis: } \\
\text { randomized controlled } \\
\text { trial. }\end{array}$ & BVS & $\begin{array}{c}\text { Kjeken et al., } \\
2013 \\
\text { NORUEGA }\end{array}$ & $\begin{array}{l}\text { Journal of } \\
\text { rehabilitation } \\
\text { medicine }\end{array}$ \\
\hline N2 & $\begin{array}{l}\text { Disease Activity, } \\
\text { Occupational } \\
\text { Participation, and Quality } \\
\text { of Life for Individuals with } \\
\text { and without Severe } \\
\text { Fatigue in Ankylosing } \\
\text { Spondylitis. }\end{array}$ & BVS & $\begin{array}{l}\text { Connolly; } \\
\text { Fitzpatrick; } \\
\text { O'Shea, 2019 } \\
\text { IRLANDA }\end{array}$ & $\begin{array}{l}\text { Occupational } \\
\text { therapy } \\
\text { international }\end{array}$ \\
\hline
\end{tabular}




\begin{tabular}{|c|c|c|c|c|}
\hline N3 & $\begin{array}{lr}\begin{array}{l}\text { Differential } \\
\text { between }\end{array} & \text { features } \\
\text { ankylosing } & \text { ppondylitis } \\
\text { and } & \text { spondylitis } \\
\text { and } & \text { associated with psoriasis } \\
\text { and inflammatory bowel } \\
\text { disease. }\end{array}$ & BVS & $\begin{array}{l}\text { Alamino et } \\
\text { al., } 2011 \\
\text { ARGENTINA }\end{array}$ & $\begin{array}{l}\text { The Journal of } \\
\text { rheumatology }\end{array}$ \\
\hline N4 & $\begin{array}{l}\text { Effects of aerobic training } \\
\text { in patients with } \\
\text { ankylosing spondylitis. }\end{array}$ & BVS & $\begin{array}{l}\text { Jennings et } \\
\text { al., } 2015 \\
\text { BRASIL }\end{array}$ & $\begin{array}{l}\text { The Journal of } \\
\text { rheumatology }\end{array}$ \\
\hline N5 & $\begin{array}{l}\text { Effects of Multidisciplinary } \\
\text { Team-Based Nurse-led } \\
\text { Transitional Care on } \\
\text { Clinical Outcomes and } \\
\text { Quality of Life in Patients } \\
\text { With Ankylosing } \\
\text { Spondylitis. A }\end{array}$ & BVS & $\begin{array}{l}\text { Liang et al., } \\
2019 \\
\text { CHINA }\end{array}$ & $\begin{array}{l}\text { Asian nursing } \\
\text { research }\end{array}$ \\
\hline N6 & $\begin{array}{l}\text { Factors related to health- } \\
\text { related quality of life in } \\
\text { ankylosing spondylitis, } \\
\text { overall and stratified by } \\
\text { sex. }\end{array}$ & BVS & $\begin{array}{l}\text { Law et al., } \\
2018 \\
\text { SUÉCIA }\end{array}$ & $\begin{array}{l}\text { Arthritis } \\
\text { research \& } \\
\text { therapy }\end{array}$ \\
\hline N7 & $\begin{array}{l}\text { Gender-attributable } \\
\text { differences in outcome of } \\
\text { ankylosing spondylitis: } \\
\text { long-term results from the } \\
\text { Outcome in Ankylosing } \\
\text { Spondylitis International } \\
\text { Study. }\end{array}$ & BVS & $\begin{array}{l}\text { Webers et al., } \\
2016 \\
\text { HOLANDA }\end{array}$ & Rheumatology \\
\hline N8 & $\begin{array}{l}\text { Impact of age, sex, } \\
\text { physical function, health- } \\
\text { related quality of life, and } \\
\text { treatment with } \\
\begin{array}{l}\text { adalimumab on work } \\
\text { status and work } \\
\text { productivity of patients } \\
\text { with ankylosing } \\
\text { spondylitis. }\end{array} \\
\end{array}$ & BVS & $\begin{array}{l}\text { Maksymowyc } \\
\text { h et al., 2010 } \\
\text { CANADÁ }\end{array}$ & $\begin{array}{l}\text { The Journal of } \\
\text { rheumatology }\end{array}$ \\
\hline N9 & $\begin{array}{l}\text { Increased body mass } \\
\text { index in ankylosing } \\
\text { spondylitis is associated } \\
\text { with greater burden of } \\
\text { symptoms and poor } \\
\text { perceptions of the } \\
\text { benefits of exercise. }\end{array}$ & BVS & $\begin{array}{l}\text { Durcan et al., } \\
2012 \\
\text { IRLANDA }\end{array}$ & $\begin{array}{l}\text { The Journal of } \\
\text { rheumatology }\end{array}$ \\
\hline
\end{tabular}




\begin{tabular}{|c|c|c|c|c|}
\hline N10 & $\begin{array}{l}\text { Relationship between } \\
\text { psychiatric status, self- } \\
\text { reported outcome } \\
\text { measures, and clinical } \\
\text { parameters in axial } \\
\text { spondyloarthritis. }\end{array}$ & BVS & $\begin{array}{l}\text { Kilic; Kilic; } \\
\text { Ozgocmen, } \\
2014 \\
\text { TURQUIA }\end{array}$ & Medicine \\
\hline N11 & $\begin{array}{l}\text { The effect of physical } \\
\text { activity and motivation on } \\
\text { function in ankylosing } \\
\text { spondylitis: a cohort } \\
\text { study. }\end{array}$ & BVS & $\begin{array}{l}\text { Brophy et al., } \\
2013 \\
\text { REINO } \\
\text { UNIDO }\end{array}$ & $\begin{array}{l}\text { Seminars in } \\
\text { arthritis and } \\
\text { rheumatism }\end{array}$ \\
\hline $\mathrm{N} 12$ & $\begin{array}{l}\text { The impact of ankylosing } \\
\text { spondylitis on female } \\
\text { sexual functions. }\end{array}$ & BVS & $\begin{array}{l}\text { Sariyildiz et } \\
\text { al., } 2013 \\
\text { TURQUIA }\end{array}$ & $\begin{array}{l}\text { International } \\
\text { journal of } \\
\text { impotence } \\
\text { research }\end{array}$ \\
\hline $\mathrm{N} 13$ & $\begin{array}{l}\text { Capacidad funcional y } \\
\text { factores asociados en } \\
\text { pacientes con espondilitis } \\
\text { anquilopoyética. }\end{array}$ & LILACS & $\begin{array}{l}\text { Figueredo et } \\
\text { al., } 2014 \\
\text { CUBA }\end{array}$ & $\begin{array}{l}\text { Revista } \\
\text { Cubana de } \\
\text { Medicina }\end{array}$ \\
\hline N14 & $\begin{array}{l}\text { Concomitância de } \\
\text { fibromialgia em pacientes } \\
\text { com } \\
\text { anquilosante. }\end{array}$ & LILACS & $\begin{array}{l}\text { Azevedo et } \\
\text { al., } 2010 \\
\text { BRASIL }\end{array}$ & $\begin{array}{c}\text { Revista } \\
\text { Brasileira de } \\
\text { Reumatologia }\end{array}$ \\
\hline N15 & $\begin{array}{l}\text { Custos diretos e indiretos } \\
\text { do tratamento de } \\
\text { pacientes com } \\
\text { espondilite anquilosante } \\
\text { pelo sistema público de } \\
\text { saúde brasileiro. }\end{array}$ & LILACS & $\begin{array}{l}\text { Azevedo et } \\
\text { al., } 2016 \\
\text { BRASIL }\end{array}$ & $\begin{array}{c}\text { Revista } \\
\text { Brasileira de } \\
\text { Reumatologia }\end{array}$ \\
\hline N16 & 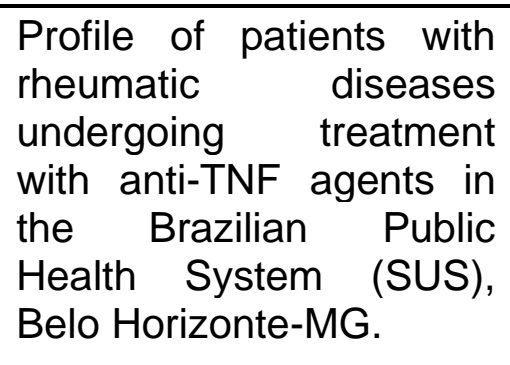 & LILACS & $\begin{array}{l}\text { Oliveira } \\
\text { Junior et al., } \\
2015 \\
\text { BRASIL }\end{array}$ & $\begin{array}{c}\text { Brazilian } \\
\text { Journal of } \\
\text { Pharmaceutica } \\
\text { I Sciences }\end{array}$ \\
\hline N17 & $\begin{array}{l}\text { Qualidade do sono em } \\
\text { pacientes } \\
\text { espondilite anquilosante. }\end{array}$ & LILACS & $\begin{array}{l}\text { Aydin et al., } \\
2015 \\
\text { TURQUIA }\end{array}$ & $\begin{array}{c}\text { Revista } \\
\text { Brasileira de } \\
\text { Reumatologia }\end{array}$ \\
\hline
\end{tabular}




\begin{tabular}{|c|c|c|c|c|}
\hline N18 & $\begin{array}{l}\text { Acute anterior uveitis as } \\
\text { a risk factor of ankylosing } \\
\text { spondylitis-a national } \\
\text { population-based study. }\end{array}$ & PUBMED & $\begin{array}{l}\text { Yen et al., } \\
2017 \\
\text { TAIWAN }\end{array}$ & $\begin{array}{l}\text { International } \\
\text { journal of } \\
\text { environmental } \\
\text { research and } \\
\text { public health }\end{array}$ \\
\hline N19 & $\begin{array}{l}\text { Association of congestive } \\
\text { heart failure and death } \\
\text { with } \\
\text { spondylitis: A nationwide } \\
\text { longitudinal cohort study } \\
\text { in Korea. }\end{array}$ & PUBMED & $\begin{array}{l}\text { Bae et al., } \\
2019 \\
\text { CORÉIA DO } \\
\text { SUL }\end{array}$ & $\begin{array}{l}\text { Journal of } \\
\text { Korean } \\
\text { Neurosurgical } \\
\text { Society }\end{array}$ \\
\hline N20 & $\begin{array}{l}\text { Impact of tumor necrosis } \\
\text { factor inhibitor versus } \\
\text { nonsteroidal } \\
\text { antiinflammatory drug } \\
\text { treatment on radiographic } \\
\text { progression in early } \\
\text { ankylosing spondylitis: its } \\
\text { relationship to } \\
\text { inflammation control } \\
\text { during treatment. }\end{array}$ & PUBMED & $\begin{array}{l}\text { Park et al., } \\
2019 \\
\text { CORÉIA DO } \\
\text { SUL }\end{array}$ & $\begin{array}{c}\text { Arthritis \& } \\
\text { Rheumatology }\end{array}$ \\
\hline
\end{tabular}

Identificaram-se duas publicações nos anos de 2010, seguida de uma em 2011; uma em 2012; três em 2013; duas em 2014; três em 2015; duas em 2016; uma em 2017; uma em 2018 e quatro publicações em 2019; quatro artigos foram desenvolvidos no Brasil e 16 em outros países como Coreia do Sul, Turquia, Irlanda, Cuba, dentre outros. Observa-se uma diversidade de periódicos, com maior frequência para o The Journal of Rheumatology (quatro artigos) e a Revista Brasileira de Reumatologia (três artigos).

\subsection{Principais debilitações físicas descritas nos artigos selecionados na amostra final}

As principais debilitações físicas advindas da EA estão descritas na Tabela 1. 
Tabela 1 - Principais debilitações físicas relatadas

\begin{tabular}{|l|l|}
\hline \multicolumn{1}{|c|}{ Debilitação Física } & \multicolumn{1}{c|}{ Relatada por } \\
\hline Dificuldades no autocuidado & $\mathrm{N} 1$ \\
\hline Diminuição da produtividade & $\mathrm{N} 1, \mathrm{~N} 2, \mathrm{~N} 8, \mathrm{~N} 13$ \\
\hline Diminuição ou prejuízo no lazer & $\mathrm{N} 1, \mathrm{~N} 2$ \\
\hline Fadiga & $\mathrm{N} 2, \mathrm{~N} 6$ \\
\hline Déficit na capacidade e mobilidade física geral & $\begin{array}{l}\mathrm{N} 1, \mathrm{~N} 2, \mathrm{~N} 3, \mathrm{~N} 4, \mathrm{~N} 6, \\
\mathrm{~N} 7, \mathrm{~N} 9, \mathrm{~N} 13\end{array}$ \\
\hline Dor articular & $\mathrm{N} 3, \mathrm{~N} 7, \mathrm{~N} 13$ \\
\hline Envolvimento do esqueleto axial & $\mathrm{N} 3$ \\
\hline $\begin{array}{l}\text { Diminuição da rotação cervical e da mobilidade espinhal } \\
\text { em si }\end{array}$ & $\mathrm{N} 3, \mathrm{~N} 13$ \\
\hline Envolvimento do quadril & $\mathrm{N} 3, \mathrm{~N} 7, \mathrm{~N} 13$ \\
\hline $\begin{array}{l}\text { Presença de sindesmófitos e/ou } \geq 3 \text { pontes intervertebrais } \\
\text { consecutivamente, na coluna cervical e/ou lombar }\end{array}$ & $\mathrm{N} 6, \mathrm{~N} 20$ \\
\hline Disfunções sexuais & $\mathrm{N} 12$ \\
\hline Sacroilíte & $\mathrm{N} 13$ \\
\hline Déficit na qualidade do sono & $\mathrm{N} 17$ \\
\hline
\end{tabular}

Outrossim, a presença destes sinais e sintomas foi relatada por outros autores (GHASEMI-RAD et al., 2015; VISHA et al., 2019; REINDERS; VAN WYK, 2012). Para N3, a Espondilite Anquilosante (EA) apresenta maior debilitação e envolvimento do esqueleto axial, observados pelos piores escores do Bath Ankylosing Spondylitis Metrological Index e do Bath Ankylosing Spondylitis Radiology Index (ALAMINO et al., 2011).

No tocante ao exame Short Form 36, alguns estudos demonstraram valores inferiores a mediana (50,0 pontos), o que confirma o prejuízo na qualidade de vida nos seguintes domínios: saúde geral (N4, N5), papel físico (N4), dor corporal (N5) e resumo do componente físico (N6) (JENNINGS et al., 2015; LIANG et al., 2019; LAW et al., 2018).

Além disso, diferenças em relação ao sexo foram verificadas: homens apresentando uma pontuação menor na função física e maior dano radiográfico, bem como mulheres com diminuição nos domínios mentais (N6) e maior frequência de entesopatias e dor articular (N7) (LAW et al., 2018; WEBERS et al., 2016).

Ademais, N2 verificou o impacto da doença na fadiga e produtividade no trabalho, relatando uma piora no rendimento empregatício, sendo as áreas da concentração, carga horária de trabalho e afazeres domésticos as principais afetadas. (CONNOLLY; FITZPATRICK; O'SHEA, 2019) 
Semelhantemente, N8 aponta pacientes com doenças concomitantes e anquilose total da coluna menos propensos a conseguir emprego, indicando os status de dor nas costas noturna, atividade da doença e qualidade de vida como fatores ligados diretamente ao status e índices de produtividade no emprego. O Presenteísmo no trabalho foi correlacionado com alguns componentes do SF-36 que avaliam a função física e dor corporal, entre outros fatores. (MAKSYMOWYCH et al., 2010)

A presença de fatores de risco como o Tabagismo (N4, N6, N7, N9, N17); Índice de Massa Corpórea (IMC) elevado (N4, N6, N9 e N20); Histórico de Uveíte Anterior (N6, N7, N9, N18); Doença Inflamatória Intestinal (N7, N9); Dislipidemia, Hipertensão Arterial Sistêmica e Diabetes Mellitus (N4, N19); Fibromialgia (N14) e a Insuficiência Cardíaca Congestiva (N19) são pertinentes na história clínica, sendo verificados em até $63,2 \%$ dos pacientes, bem como em outros estudos (N1) (KJEKEN et al., 2013; JENNINGS et al., 2015; LAW et al., 2018; WEBERS et al., 2016; DURCAN et al., 2012; AYDIN et al., 2015; YEN et al., 2017; AZEVEDO et al., 2010; BAE et al., 2019; PARK et al., 2019; VISHA et al., 2019; REINDERS; VAN WYK, 2012).

Pesquisa chinesa observou prevalência de Síndrome Metabólica, relacionando a doença aos fatores de riscos (LIU et al., 2019). Porém, problemas cardíacos são apontados como menos comuns por outros autores (VISHA et al., 2019). Além disso, conforme N9, o IMC elevado correlaciona com maior índice de atividade da doença, dor noturna e nas costas, rigidez e fadiga (DURCAN et al., 2012).

Por conseguinte, conforme N2, a fadiga interfere negativamente na participação social, podendo justificar futuros problemas psicológicos. Ansiedade e depressão são os principais analisados, predominando a ansiedade em homens (N10) e ambos os transtornos em pacientes mais velhos, com má função e qualidade de vida (N11) (CONNOLLY; FITZPATRICK; O'SHEA, 2019; KILIC; KILIC; OZGOCMEN, 2014; BROPHY et al., 2013).

Similarmente, as debilitações têm influenciado negativamente nas funções sexuais femininas (N12) e no sono, analisado por meio do exame Bath Ankylosing Spondylitis Activity Index (N17). Outros autores discutem o bemestar dos pacientes frente ao envelhecimento, apontando a necessidade de 
tratamento personalizado (MARTINDALE; KASHEFI; GOODACRE, 2016; SARIYILDIZ et al., 2013; AYDIN et al., 2015).

Em contrapartida, observou-se a importância do gerenciamento de fadiga pelos portadores. Para N2, essas ferramentas possibilitam aos participantes conviverem com a doença sem gerar grandes prejuízos no meio social e laboral, como a criação de rotinas, o diálogo com familiares e colegas de trabalho (CONNOLLY; FITZPATRICK; O'SHEA, 2019).

Nesse cenário, a prática de exercícios auxilia na manutenção da função física e qualidade de vida, porém com baixa aceitabilidade; conforme N11, o prejuízo funcional e a prática de atividade física eram inversamente proporcionais. Além disso, participantes com maior nível de motivação demonstraram melhoria na função física, mesmo com a alta atividade da doença (BROPHY et al., 2013).

Pesquisadores apontam a gravidade da dor como um determinante para a persistência no tratamento, sendo um fenômeno comum a cinesiofobia (OSKAY et al., 2017). Entretanto, a colaboração profissional tem gerado questionamentos. Em N2, a maioria dos participantes relataram falta de discussão sobre o gerenciamento da fadiga, tanto pelos agentes de saúde quanto pelos pacientes, o que explica o autodesenvolvimento de medidas de controle da rigidez e dor (CONNOLLY; FITZPATRICK; O'SHEA, 2019).

\subsection{Prevalência da Espondilite Anquilosante e perfil epidemiológico, conforme sexo, idade de início dos sintomas e sintomatologia}

Como observado na Tabela 2, as médias de idade dos pacientes não possuem grande discrepância, estando condizentes com outras literaturas como Gouveia, Elmann e Morales (2012) que afirmam que a doença, quando de início em adultos, manifesta-se os 20 e 30 anos 
Tabela 2- Médias de idade de pacientes com espondilite anquilosante por artigo

\begin{tabular}{ccc}
\hline $\begin{array}{c}\text { IDENTIFICAÇÃO } \\
\text { DO ARTIGO }\end{array}$ & MÉDIA DE IDADE (ANOS) & $\begin{array}{c}\text { TAMANHO DA } \\
\text { AMOSTRA }\end{array}$ \\
\hline N1 & 49 & 95 \\
N2 & 46,5 & 50 \\
N3 & 42 & 1072 \\
N4 & 42,9 & 35 \\
N5 & 29,2 & 49 \\
N6 & 49 & 210 \\
N7 & 45,1 & 216 \\
N8 & Não se aplica & Não se aplica \\
N9 & 45,1 & 46 \\
N10 & 36,3 & 316 \\
N11 & Não se aplica & Não se aplica \\
N12 & 34,1 & 37 \\
N13 & 40,1 & 80 \\
N14 & 43,7 & 71 \\
N15 & 43,6 & 93 \\
N16 & 44,2 & 55 \\
N17 & 43 & 55 \\
N18 & Não se aplica & Não se aplica \\
N19 & 40,2 & 12988 \\
N20 & Não se aplica & Não se aplica \\
\hline
\end{tabular}

N7 observou que, apesar dos primeiros sintomas se manifestarem, em média, a partir de 23,2 anos de idade, há demora no diagnóstico de aproximadamente 9 anos (WEBERS et al., 2016). A partir da análise de outros estudos verificaram-se atrasos de 7,9 (FALLAHI; JAMSHIDI, 2016), 6,7 (NAKASHIMA et al., 2015) e 8,7 anos (BANDINELLI et al., 2016), constatando que essa problemática é frequente. Em contraponto, Nakashima et al. (2015) e Sorensen e Hetland (2015) verificaram um decréscimo no atraso desde os anos 2000, devido a maior conscientização sobre a EA.

Nesse sentido, o diagnóstico tardio resulta em piora do prognóstico por meio de maior atividade da doença, aumento da fadiga e diminuição da mobilidade dos quadris e da coluna (FALLAHI e JAMSHIDI, 2016). Entretanto, o perfil do paciente também afeta na demora, sendo a menor escolaridade, 0 trabalho manual e HLA-B27 negativo os principais fatores (BANDINELII et al., 2016). A obrigatoriedade de alterações radiográficas segundo os critérios de Nova lorque também influencia no retardo diagnóstico, uma vez que tais 
alterações só se manifestam após anos do início da doença (SORENSEN e HETLAND, 2015).

Quanto à raça/cor/etnia, N13 e N16 indicaram que a mais afetada pela doença é a branca (FIGUEREDO et al., 2014; OLIVEIRA JÚNIOR et al., 2015). Esse fato aparenta ter relação com a prevalência do antígeno HLA-B27 entre caucasianos, podendo influenciar na precocidade da EA e maior ocorrência familiar, fatores menos observados em pacientes HLA-B27 negativos (ARÉVALO et al., 2018; YANG et al., 2013). Sob outra perspectiva, Ribeiro et al. (2016), identificou que a cor negra apresenta menores escores de qualidade de vida, podendo ter como causas fatores genéticos ou socioeconômicos.

Do total de artigos, 12 (N1, N2, N3, N6, N7, N9, N13, N14, N15, N16, N17, N19) possibilitaram a análise da população conforme o sexo, observandose maior prevalência do sexo masculino (KJEKEN et al., 2013; CONNOLLY; FITZPATRICK; O'SHEA, 2019; ALAMINO et al., 2011; LAW et al., 2018; WEBERS et al., 2016; DURCAN et al., 2012; FIGUEREDO et al., 2014; AZEVEDO et al., 2010; AZEVEDO et al., 2016; OLIVEIRA JUNIOR et al., 2015; AYDIN et al., 2015; BAE et al., 2019). Apesar de alguns dos estudos, como N13 e N16 (FIGUEREDO et al., 2014; OLIVEIRA JÚNIOR et al., 2015), utilizarem amostras pequenas e de conveniência, os resultados encontram-se de acordo com outras literaturas, como Duarte et al. (2014) e Gouveia, Elmann e Morales (2012).

N16 apontou que homens apresentam melhor status funcional que mulheres, correlacionando melhores níveis de funcionalidade (HAQ-DI) a melhores índices de qualidade de vida e de atividade da doença (BASDAI e CDAI) (OLIVEIRA JÚNIOR et al., 2015). Da mesma forma, N6 identifica que mulheres tendem a relatar pior função física e mental (SF-36) devido à doença (LAW et al., 2018).

Em contraponto, apesar de N7 apontar piores índices de qualidade de vida (ASQOL) entre mulheres, foi atribuído devido a diferentes percepções da dor e não necessariamente a fatores biológicos, não havendo discrepâncias entre os sexos quanto às funções físicas e atividade da doença (WEBERS et al., 2016).

Entretanto, é fato a existência de diferenças físicas entre sexos quanto a atividade da EA. De tal maneira, em homens observou-se presença de danos 
radiográficos mais intensos (N7), além de maior IMC em relação a pacientes de sexo feminino (N6), predispondo pior impacto da doença e mais danos a coluna (WEBERS et al., 2016; LAW et al., 2018; DURCAN et al., 2012; DEMINGER et al., 2018). Em comparação, conforme N6, a fadiga é mais presente entre as mulheres, trazendo piores consequências no aspecto social e profissional e limitações das atividades cotidianas (LAW et al., 2018).

\subsection{Tratamento e acompanhamento multiprofissional na qualidade de vida dos pacientes diagnosticados com EA}

Do total de 20 artigos analisados, 13 (N8, N16, N20, N6, N17, N3, N7, N12, $\mathrm{N} 15, \mathrm{~N} 1, \mathrm{~N} 4, \mathrm{~N} 11, \mathrm{~N} 5)$ possibilitaram correlacionar o tratamento à qualidade de vida. Mormente, essa correlação estrutura-se em duas linhas: o consumo de medicamentos e a prática de exercícios, bem como a associação de ambos.

Observou-se o predomínio no uso das seguintes classes de medicamentos: Inibidores do Fator de Necrose Tumoral (TNF-i), os Medicamentos Modificadores do Curso da Doença (MMCD's) e os antiinflamatórios não esteroides (AINEs) (OLIVEIRA JUNIOR et al., 2015; PARK et al., 2019).

A terapia com os TNF-i é uma das principais relatada nos estudos (N8 e N16), com destaque para os agentes Adalimumabe ${ }^{\circledR}$, Etanercepete ${ }^{\circledR}$ e Infliximabe® (MAKSYMOWYCH et al., 2010; OLIVEIRA JÚNIOR et al., 2015). De fato, o uso desta classe medicamentosa apresenta resultados positivos para o bem-estar dos pacientes, sem efeitos colaterais graves, conforme N8 e N20 (MAKSYMOWYCH et al., 2010; PARK et al., 2019).

Outro estudo relata maior persistência no tratamento com TNF-i's, de aproximadamente 80\%, em relação MMCD's (MACHADO et al., 2016). Ademais, N20 verificou menor progressão radiográfica no início da doença com o uso dos anti-TNF's (PARK et al., 2019).

Entretanto, no estudo N6, apenas 1/5 dos participantes utilizavam TNFi's, sendo mais expressiva a associação destes com os MMCD's (34,8\%) (LAW et al., 2018). Semelhantemente, quase metade dos participantes de N17 administravam MMCD's $(40,7 \%)$, seguido dos AINE's $(29,6 \%)$ e dos TNF-i's $(20,4 \%)$ (AYDIN et al., 2015). 
Da mesma forma, o predomínio do uso dos AINE's também foi verificado, seja isoladamente (N3,N7), seja em associação com fármacos antimicrobianos ou com anti-TNF's (N12) (ALAMINO et al., 2011; WEBERS et al., 2016; SARIYILDIZ et al., 2013).

Em relação ao consumo de Adalimumabe ${ }^{\circledR}$, N8 retrata a manutenção da produtividade e do emprego de pacientes com EA, apresentando bons índices no exame Work Productivity and Activity Impairment Questionnaire-Specific Health Problem, com diminuição do presenteísmo e absenteísmo (MAKSYMOWYCH et al., 2010). Outro estudo verificou melhora na mobilidade vertebral, na atividade da doença, função física e no exame Ankylosing Spondylitis Quality of Life, significando um avanço na qualidade de vida (VAN DER HEIJDE et al., 2015).

Resultados semelhantes foram observados em uma pesquisa com 963 pacientes utilizando o Golimumabe ${ }^{\circledR}$ ou o Infliximabe $\AA$, evidenciando melhora na condição física e na manutenção do status de emprego (CLAUDEPIERRE et al., 2019).

N16 observou a supremacia do consumo de Adalimumabe ${ }^{\circledR}$ entre pacientes com EA pelo Sistema Único de Saúde (SUS), alcançando um percentual de cerca de $72 \%$ do total, seguido do Etanercepete ${ }^{\circledR}$, com valor inferior a 20\% (OLIVEIRA JUNIOR et al., 2015).

No tocante ao uso de Golimumabe®, estudos indicam uma redução nos distúrbios do sono de pacientes com EA, com melhora tanto no Exame Jenkins Sleep Evaluation Questionnaire, quanto no SF-36, dor nas costas total e noturna (DEODHAR et al., 2010). O efeito também foi avaliado por Van Der Heijde et al. (2014) apresentando melhora na qualidade de vida e na produtividade do trabalho. Igualmente, Krüger et al. (2019) constatou diminuição da fadiga e melhorias na capacidade funcional e qualidade de vida.

Dessa forma, o uso dos Inibidores do TNF apresenta-se como uma ferramenta ímpar na diminuição da dor e manutenção da função física em EA. Ademais, N15 constatou maior gasto público com o tratamento de EA entre 2011 e 2012, porém com baixo investimento em fisioterapia, evidenciando crescimento de medicamentos como terapêutica para a doença (AZEVEDO et al., 2016). 
Além disso, observou-se que a associação da terapia com exercícios físicos melhora o quadro funcional e a qualidade de vida. Conforme N1, programas de reabilitação voltados para a prática de exercícios apresentaram melhora significativa nos quesitos vitalidade, dor corporal e funções física e mental do Questionário de Qualidade de Vida SF-36 (KJEKEN et al., 2013).

Semelhantemente, N4 observou a influência dos exercícios aeróbicos no tratamento, proporcionando uma melhor função física e capacidade pulmonar, mas sem alteração significativa na qualidade de vida, diferentemente do constatado por N1 (KJEKEN et al., 2013; JENNINGS et al., 2015).

Outrossim, N11 analisou a motivação como estimulador para o tratamento, constatando melhoria na função em pacientes com alta atividade da doença, relacionando o acompanhamento psicológico como fator de persistência e sucesso na terapia (BROPHY et al., 2013). Concomitantemente, resultados de estudo sobre motivação e apoio para atividade física indicam melhoria da qualidade de vida e da mobilidade espinhal (O'DWYER et al., 2017).

Ademais, N5 avaliou a influência de uma equipe multidisciplinar e constatou melhoras significativas em aspectos clínicos da doença, bem como na qualidade de vida. Entretanto, uma pesquisa sobre a fisioterapia em quatro pacientes com EA revelou diferenças não significativas na qualidade de vida, mas aumento da mobilidade espinhal (ROSIN et al., 2017).

\section{Conclusão}

A literatura pesquisada revelou que a Espondilite Anquilosante influencia amplamente na qualidade de vida de pessoas acometidas por esta patologia, sendo um dos principais motivos seu caráter incapacitante.

Nesse sentido, faz-se relevante pontuar que tais consequências são dependentes do perfil do paciente em detrimento de fatores como sexo, hábitos e nível socioeconômico, o que intervém tanto na forma como a doença se expressa como na possibilidade de uma abordagem terapêutica precoce.

Devido a essa multifatoriedade da EA, constata-se a relevância de um tratamento multiprofissional e eficaz, que combina o uso de fármacos com a prática de atividade física, garantindo a manutenção de uma rotina saudável para o paciente, item de grande importância tendo em vista o potencial da 
doença em acarretar danos econômicos aos enfermos devido à idade em que se estabelece e à possibilidade de incapacidade laboral.

\section{$5 \quad$ Referências}

ALAMINO, R. P. et al. Differential features between primary ankylosing spondylitis and spondylitis associated with psoriasis and inflammatory bowel disease. The Journal of rheumatology, v. 38, n. 8, p. 1656-1660, 2011.

ARAÚJO, C. A. et al. Tratamento fisioterapêutico em Espondilite Anquilosante: uma revisão sistemática. UNILUS Ensino e Pesquisa, v. 12, n. 29, p. 53-58, 2015.

ARAÚJO, H. G. C. Aspectos nutricionais na terapêutica da Espondilite Anquilosante: Uma revisão da literatura. 2017. $37 \mathrm{f}$. Trabalho de Conclusão de Curso (Graduação em Nutrição) - Universidade Federal do Rio Grande do Norte, 2017.

ARÉVALO, M. et al. Influence of HLA-B27 on the Ankylosing Spondylitis phenotype: results from the REGISPONSER database. Arthritis Research \& Therapy, v. 20, n. 1, p. 1-6, 2018.

AYDIN, E. et al. Qualidade do sono em pacientes com espondilite anquilosante. Revista Brasileira de Reumatologia, v. 55, n. 4, p. 340-345, 2015.

AZEVEDO, V. F. et al. Concomitância de fibromialgia em pacientes com espondilite anquilosante. Revista Brasileira de Reumatologia, v. 50, n. 6, p. 646-650, 2010.

AZEVEDO, V. F. et al. Custos diretos e indiretos do tratamento de pacientes com espondilite anquilosante pelo sistema público de saúde brasileiro. Revista Brasileira de Reumatologia, v. 56, n. 2, p. 131-137, 2016.

BAE, K. H. et al. Association of congestive heart failure and death with ankylosing spondylitis: a nationwide longitudinal cohort study in Korea. Journal of Korean Neurosurgical Society, v. 62, n. 2, p. 217, 2019.

BANDINELLI, F. et al. Impact of gender, work, and clinical presentation on diagnostic delay in Italian patients with primary ankylosing spondylitis. Clinical rheumatology, v. 35, n. 2, p. 473-478, 2016.

BOTELHO, L. L. R.; CUNHA, C. C. A.; MACEDO, M. O método da revisão integrativa nos estudos organizacionais. Gestão e sociedade, v. 5, n. 11, p. 121-136, 2011.

BRASIL. Ministério da Saúde. Secretaria de Atenção à Saúde; Secretaria de Ciência, Tecnologia e Insumos Estratégicos. Portaria Conjunta № 25, de 22 de outubro de 2018. Aprova o Protocolo Clínico e Diretrizes Terapêuticas da Espondilite Ancilosante. Diário Oficial da União, Brasília, DF, 26 de out. 2018. p. 58.

BROPHY, S. et al. The effect of physical activity and motivation on function in ankylosing spondylitis: a cohort study. In: Seminars in arthritis and rheumatism. WB Saunders, 2013. p. 619-626.

CLAUDEPIERRE, P. et al. Treatment with golimumab or infliximab reduces health resource utilization and increases work productivity in patients with ankylosing spondylitis in the QUO-VADIS study, a large, prospective real-life cohort. International journal of rheumatic diseases, v. 22, n. 6, p. 995-1001, 2019. 
COMISSÃO DE ESPONDILOARTRITES. Espondilite anquilosante: cartilha para pacientes. Sociedade Brasileira de Reumatologia, 2012.

CONNOLLY, D.; FITZPATRICK, C.; O'SHEA, F. Disease activity, occupational participation, and quality of life for individuals with and without severe fatigue in ankylosing spondylitis. Occupational therapy international, v. 2019, 2019.

DEMINGER, A. et al. A five-year prospective study of spinal radiographic progression and its predictors in men and women with ankylosing spondylitis. Arthritis research \& therapy, v. 20, n. 1, p. 162, 2018.

DEODHAR, A. et al. Golimumab reduces sleep disturbance in patients with active ankylosing spondylitis: results from a randomized, placebo-controlled trial. Arthritis Care \& Research, v. 62, n. 9, p. 1266-1271, 2010.

DUARTE, A. P. et al. Perfil epidemiológico da espondiloartrite de início juvenil comparada com a espondiloartrite de início na vida adulta em uma grande coorte brasileira. Revista Brasileira de Reumatologia, v. 54, n. 6, p. 424-430, 2014.

DURCAN, L. et al. Increased body mass index in ankylosing spondylitis is associated with greater burden of symptoms and poor perceptions of the benefits of exercise. The Journal of rheumatology, v. 39, n. 12, p. 2310-2314, 2012.

FALLAHI, S.; JAMSHIDI, A. R. Diagnostic delay in ankylosing spondylitis: related factors and prognostic outcomes. Archives of rheumatology, v. 31, n.1, p. 24, 2016.

FIGUEREDO, N. et al. Capacidad funcional y factores asociados en pacientes con espondilitis anquilopoyética. Revista Cubana de Medicina, v. 53, n. 1, p. 50-60, 2014.

GHASEMI-RAD, M. et al. Ankylosing spondylitis: a state of the art factual backbone. World Journal of Radiology, v. 7, n. 9, p. 236, 2015.

GOUVEIA, E.B.; ELMANN, D.; MORALES, M.S.A. Espondilite Anquilosante e uveíte: revisão. Revista Brasileira de Reumatologia, v. 52, n. 5, p. 749-756, 2012.

JENNINGS, F. et al. Effects of aerobic training in patients with ankylosing spondylitis. The Journal of rheumatology, v. 42, n. 12, p. 2347-2353, 2015.

KILIC, G.; KILIC, E.; OZGOCMEN, S. Relationship between psychiatric status, selfreported outcome measures, and clinical parameters in axial spondyloarthritis. Medicine, v. 93, n. 29, 2014.

KJEKEN, I. et al. A three-week multidisciplinary in-patient rehabilitation programme had positive long-term effects in patients with ankylosing spondylitis: randomized controlled trial. Journal of rehabilitation medicine, v. 45, n. 3, p. 260-267, 2013.

KRÜGER, K. et al. Patient-reported outcomes with golimumab in patients with rheumatoid arthritis, psoriatic arthritis, and ankylosing spondylitis: non-interventional study GO-NICE in Germany. Rheumatology international, v. 39, n. 1, p. 131-140, 2019.

LAW, L. et al. Factors related to health-related quality of life in ankylosing spondylitis, overall and stratified by sex. Arthritis research \& therapy, v. 20, n. 1, p. 1-12, 2018. 
LIANG, L. et al. Effects of multidisciplinary team-based nurse-led transitional care on clinical outcomes and quality of life in patients with ankylosing spondylitis. Asian nursing research, v. 13, n. 2, p. 107-114, 2019.

LIU, M. et al. Prevalence of metabolic syndrome and its associated factors in Chinese patients with ankylosing spondylitis. Diabetes, Metabolic Syndrome and Obesity: Targets and Therapy, v. 12, p. 477, 2019.

MACHADO, M. A. A. et al. Persistência do tratamento em pacientes com artrite reumatoide e espondilite anquilosante. Revista de Saúde Pública, v. 50, p. 50, 2016.

MAKSYMOWYCH, W. P. et al. Impact of age, sex, physical function, health-related quality of life, and treatment with adalimumab on work status and work productivity of patients with ankylosing spondylitis. The Journal of rheumatology, v. 37, n. 2, p. 385392, 2010.

MARTINDALE, J.; KASHEFI, E.; GOODACRE, L. An Exploratory Study of the Experience of Ageing with Ankylosing Spondylitis:'Same Backdrop but a Changing Scene'. Musculoskeletal Care, v. 14, n. 3, p. 156-165, 2016.

NAKASHIMA, Y. et al. Delayed diagnosis of ankylosing spondylitis in a Japanese population. Modern rheumatology, v. 26, n. 3, p. 421-425, 2015.

O'DWYER, T. et al. Behaviour change intervention increases physical activity, spinal mobility and quality of life in adults with ankylosing spondylitis: a randomised trial. Journal of physiotherapy, v. 63, n. 1, p. 30-39, 2017.

OLIVEIRA JUNIOR, H. A. et al. Profile of patients with rheumatic diseases undergoing treatment with anti-TNF agents in the Brazilian Public Health System (SUS), Belo Horizonte-MG. Brazilian Journal of Pharmaceutical Sciences, v. 51, n. 3, p. 709719, 2015.

OSKAY, D. et al. Relationship between kinesiophobia and pain, quality of life, functional status, disease activity, mobility, and depression in patients with ankylosing spondylitis. Turkish Journal of Mmedical Sciences, v. 47, n. 5, p. 1340-1347, 2017.

PARK, J. W. et al. Impact of tumor necrosis factor inhibitor versus nonsteroidal antiinflammatory drug treatment on radiographic progression in early ankylosing spondylitis: its relationship to inflammation control during treatment. Arthritis \& Rheumatology, v. 71, n. 1, p. 82-90, 2019.

REINDERS, A.; VAN WYK, M. J. Bamboo spine-X-ray findings of ankylosing spondylitis revisited. SA Journal of Radiology, v. 16, n. 3, 2012.

RIBEIRO, S. L. E. et al. Qualidade de vida nas espondiloartrites: análise de uma grande coorte brasileira. Revista Brasileira de Reumatologia [online]. 2016. Vol. 56, n.1, pp. 22-27. ISSN 1809-4570.

RODRIGUES, C. E. M. et al. Baixa prevalência das manifestações extra-articulares renais, cardíacas, pulmonares e neurológicas nas espondiloartrites: análise do Registro Brasileiro de Espondiloartrites. Revista Brasileira de Reumatologia, v. 52, n. 3, p. 379-383, 2012.

ROSIN, F. et al. Intervenção fisioterapêutica em indivíduos com espondilite anquilosante. Arquivos de Ciências da Saúde, v. 24, n. 2, p. 19-24, 2017. 
SAMPAIO-BARROS, P. D. et al. Recomendações sobre o diagnóstico e tratamento da espondilite anquilosante. Revista Brasileira de Reumatologia, v. 53, p. 242-257, 2013.

SARIYILDIZ, M. A. et al. The impact of ankylosing spondylitis on female sexual functions. International journal of impotence research, v. 25, n. 3, p. 104-108, 2013.

SORENSEN, J.; HETLAND, M. L. Diagnostic delay in patients with rheumatoid arthritis, psoriatic arthritis and ankylosing spondylitis: results from the Danish nationwide

DANBIO registry. Annals of the rheumatic diseases, v. 74, n. 3, p. e12-e12, 2015.

VAN DER HEIJDE, D. et al. Maintenance of improvement in spinal mobility, physical function and quality of life in patients with ankylosing spondylitis after 5 years in a clinical trial of adalimumab. Rheumatology, v. 54, n. 7, p. 1210-1219, 2015.

VAN DER HEIJDE, D. et al. The effect of golimumab therapy on disease activity and health-related quality of life in patients with ankylosing spondylitis: 2-year results of the GO-RAISE trial. The Journal of rheumatology, v. 41, n. 6, p. 1095-1103, 2014.

VISHA, M. G. et al. A review on ankylosing spondylitis. International Journal of Clinicopathological Correlation, v. 3, n. 2, p. 44, 2019.

WEBERS, C. et al. Gender-attributable differences in outcome of ankylosing spondylitis: long-term results from the Outcome in Ankylosing Spondylitis International Study. Rheumatology, v. 55, n. 3, p. 419-428, 2016.

YANG, M. et al. Epidemiological comparison of clinical manifestations according to HLA-B $^{*} 27$ carrier status of Chinese Ankylosing Spondylitis patients. Tissue Antigens, v. 82, n. 5 , p. 338-343, 2013.

YEN, J. et al. Acute anterior uveitis as a risk factor of ankylosing spondylitis-a national population-based study. International journal of environmental research and public health, v. 14, n. 1, p. 107, 2017. 